\title{
Parallel Pencil-Beam Redefinition Algorithm
}

\author{
Paul Alderson ${ }^{1}$, Mark Wright ${ }^{1}$, Amit Jain ${ }^{1}$, and Richard Boyd ${ }^{2}$ \\ 1 Department of Computer Science \\ Boise State University \\ Boise, Idaho 83725, USA \\ \{mwright, aalderso\}@onyx.boisestate.edu, amit@cs.boisestate.edu \\ 2 MD Anderson Cancer Center \\ University of Texas \\ 1515 Holcombe Blvd, Houston, TX 77030, USA \\ rboyd@mdanderson.org
}

\begin{abstract}
The growing sophistication in radiation treatment strategies requires the utilization of increasingly accurate, but computationally intense, dose algorithms, such as the electron pencil-beam redefinition algorithm (PBRA). The sequential implementation of the PBRA is in production use at the MD Anderson Cancer center. The PBRA is difficult to parallelize because of the large amounts of data involved that is accessed in an irregular pattern taking varying amounts of time in each iteration. A case study of the parallelization of the PBRA code on a Beowulf cluster using PVM and PThreads is presented. The solution uses a non-trivial way of exchanging minimal amount of data between processes to allow a natural partitioning to work. Multi-threading is used to cut down on the communication times between CPUs in the same box. Finally, an adaptive load-balancing technique is used to further improve the speedup.
\end{abstract}

\section{Introduction}

Radiation therapy was one of the first medical disciplines where computers were used to aid the process of planning treatments; the first paper on the use of computers to calculate radiation dose distributions appeared almost 50 years ago [1]. Today, computers are involved in practically all areas of radiation therapy. Treatment planning functions include image-based tumor localization, image segmentation, virtual therapy simulation, dose calculation, and optimization. Treatment delivery functions include controlling delivery systems and treatment verification. The growing sophistication in radiation treatment strategies requires the utilization of increasingly accurate, but computationally intense, dose algorithms, such as the electron pencil-beam redefinition algorithm (PBRA) [2]. The demanding pace of the radiation therapy clinic requires the employment of these advanced dose algorithms under the most optimum conditions available in terms of computation speed. Parallel processing using multi-computer clusters or multi-processor platforms are now being introduced to the clinic for this 
purpose, and the PBRA, with its extensive use of multi-dimensional arrays, is a good candidate for parallel processing.

In Section 2, we present an analysis of the PBRA sequential code. In Section 3, we describe the parallelization of the PBRA code as a series of refinements that were implemented along with the results obtained after each refinement. Section 4 further discusses some properties of the current parallel implementation. Finally, in Section 5, some conclusions are presented.

\section{Sequential Code}

The PBRA algorithm was implemented in FORTRAN by Robert Boyd in 1999. It is in production use at the University of Texas MD Andersen Cancer Center. We will highlight some features of the PBRA code that are relevant to the parallel implementation.

The PBRA code uses 16 three-dimensional arrays and several other lower dimensional arrays. The size of the arrays is about $45 \mathrm{MB}$. The inner core of the code is the function pencil_beam_redefinition(), which has a triply-nested loop that is iterated several times. Profiling shows that this function takes up about $99.8 \%$ of the total execution time. The following code sketch shows the structure of the pencil_beam_redefinition() function.

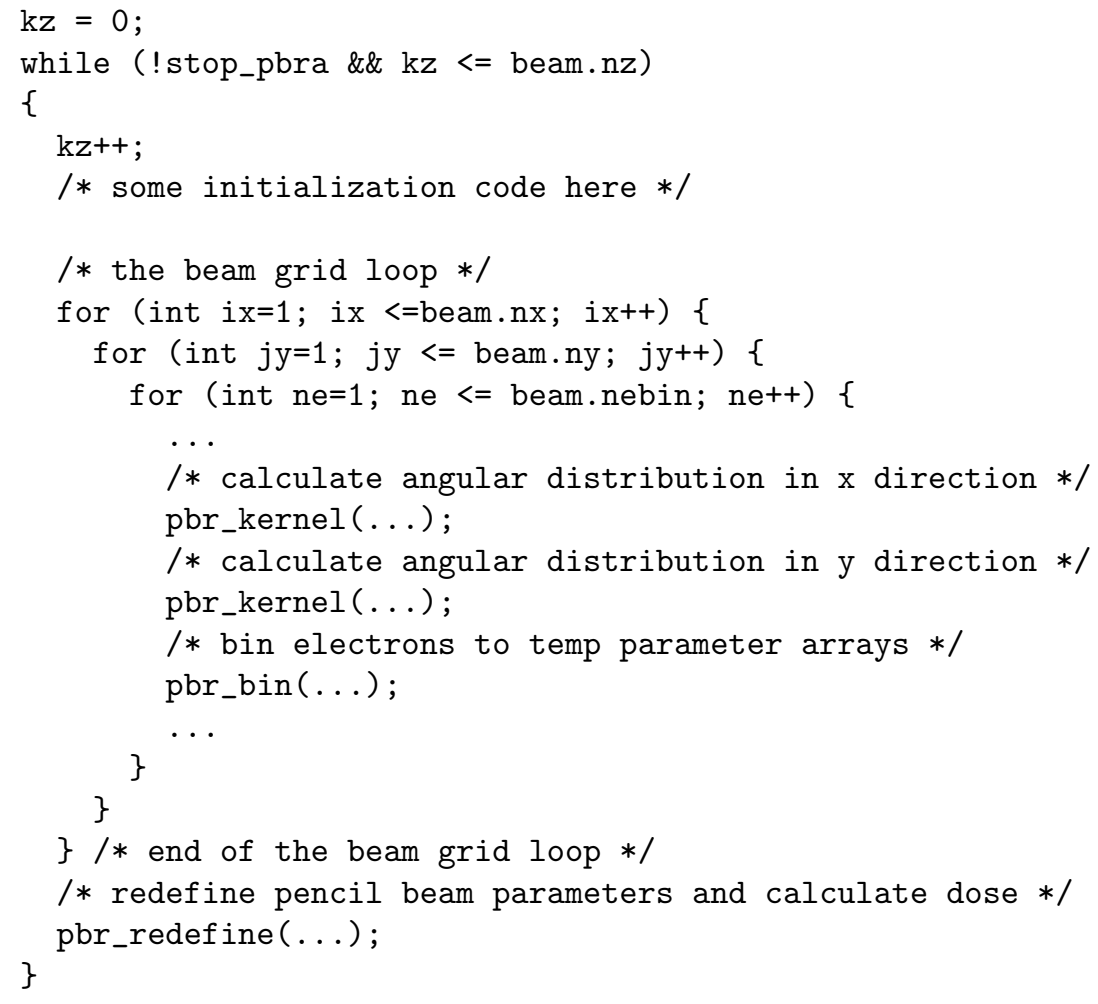


The main loop of pencil_beam_redefinition() also contains some additional straight-line code that takes constant time and some function calls that perform binary search. However these take an insignificant amount of time and are hence omitted from the sketch shown above. Currently, the code uses beam.nx $=91$, beam $. \mathrm{ny}=91$, beam $. \mathrm{nz}=25$, and nebin $=25$. Profiling on sample data shows that the functions pbr_kernel() and pbr_bin() take up about $97.6 \%$ of the total execution time.

The function pbr_kernel() takes linear time $(O$ (beam.nx) or $O$ (beam.ny)). The pbr_kernel() function computes new ranges in $x$ and $y$ direction that are used in the pbr_bin() function to update the main three -dimensional arrays. The execution time for pbr_bin( $)$ is dominated by a doubly-nested loop that is $O((x \max -x \operatorname{xin}+1) \times(y \max -\mathrm{ymin}+1))$, where the parameters xmin, $x \max$, ymin, ymax are computed in the two invocations of the pbr_kernel() function. The function pbr_bin() function updates the main three-dimensional arrays in an irregular manner, which makes it difficult to come up with a simple partitioning scheme for parallel implementation.

\section{Parallelization of PBRA}

Initially, the PBRA code was rewritten in $\mathrm{C} / \mathrm{C}++$. The $\mathrm{C} / \mathrm{C}++$ version is used as the basis for comparing performance. During this section, we will discuss timing results that are all generated using the same data set. For this data set, the sequential PBRA code ran in 2050 seconds. In production use, the PBRA code may be used to run on several data sets in a sequence. In the next section, we will present results for different data sets.

The sequential timing skips the initialization of data structures and the final writing of the dosage data to disk. These two take insignificant amount of time relative to the total running time. Before we describe the parallel implementation, we need to describe the experimental setup.

\subsection{Experimental Setup}

A Beowulf-cluster was used for demonstrating the viability of parallel PBRA code. The cluster has 6 dual-processor $166 \mathrm{MHz}$ Pentium PCs, each with $64 \mathrm{MB}$ of memory, connected via a $100 \mathrm{MBits} / \mathrm{s}$ Ethernet hub.

The PCs are running RedHat Linux 7.1 with a version 2.4.2-2 SMP kernel. The $\mathrm{C} / \mathrm{C}++$ compiler used is the $\mathrm{GNU} \mathrm{C} / \mathrm{C}++$ compiler version 2.96 with the optimizer option enabled. PVM version 3.4.3 and XPVM version 1.2.5 [3] are being used. For threads, the native POSIX threads library in Linux is being used.

\subsection{Initial PVM implementation}

The parallel implementation uses the PVM Master/Slave model. The master process initializes some data structures, spawns off the PVM daemon, adds machines to PVM, spawns off slave processes, multicasts some data structures to 
the slave processes and then waits to get the dosage arrays back from the slave processes. Embedding PVM makes it transparent to the users.

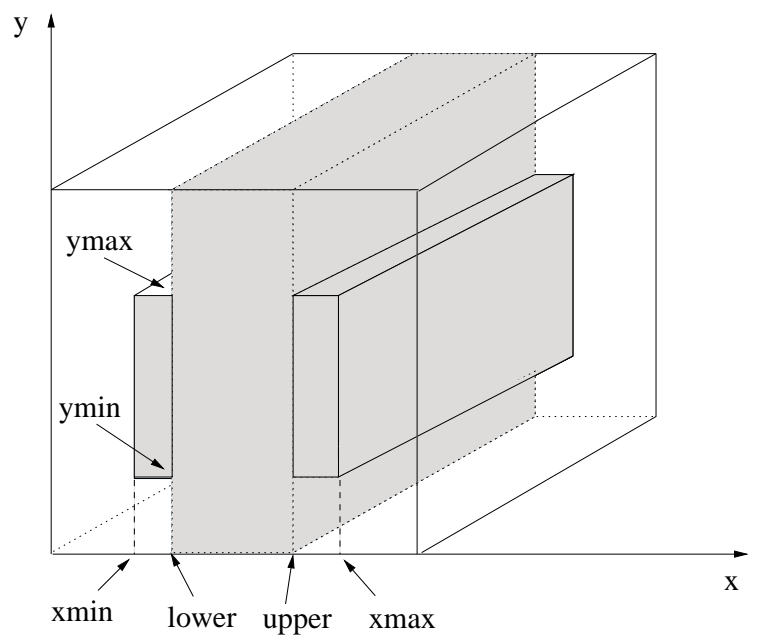

Fig. 1. Spreading of a beam being processed in one slice by one process

The main loop in pencil_beam_redefinition is being run by each slave process. However, each process works on one slice of the main three-dimensional arrays. The slicing was chosen to be done in the $X$-axis. If we have $p$ processes and $n$ is the size in the $X$ dimension, then each process is responsible for a threedimensional slice of about $n / p$ width in the $X$ axis. However, as each process simulates the beam through its slice, the beam may scatter to other slices. See Figure 1 for an illustration. Most of the time the scattering is to adjoining slices but the scattering could be throughout. One effect of this observation is that each slave process has to fully allocate some of the three-dimensional arrays even though they only work on one slice.

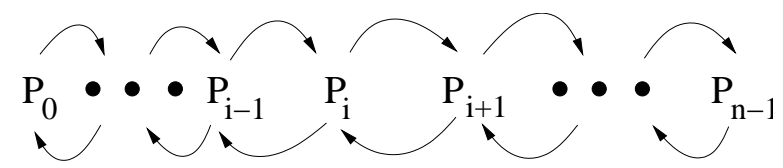

Fig. 2. Compensation for beam scattering at the end of each outer iteration

To solve the problem of beam-scattering, the processes exchange partial amounts of data at the end of each iteration (which also synchronizes the processes). The communication pattern is shown in Figure 2. Each process $P_{i}$ receives appropriate parts of the three-dimensional arrays from $P_{i-1}$ as well as 
$P_{i+1}$. Each process $P_{i}$ combines the received data with its computed data. Then $P_{i}$ sends the appropriate parts of the data off to its two neighbors. The processes $P_{0}$ and $P_{n-1}$ bootstrap the process, one in each direction. The amount of data exchanged is dependent upon how much the beam scatters.

After some more fine-tuning, the best timing with the initial implementation was 657 seconds with 12 slave processes, for a speedup of 3.12 .

\subsection{Using PThreads on Each Machine}

Using XPVM the communication patterns of the PBRA parallel code was observed. It was realized that the communication time can be reduced by letting only one process run per machine but use two threads (since each machine was a SMP machine with 2 CPUs). The main advantage was that threads would share the memory, so no communication would be required.

Each thread is now running the entire triply-nested for loop. To obtain a better load balance, the threads are assigned iterations in a round-robin fashion. Another problem is the need to synchronize threads in between calls to pbr_kernel and for the call to pbr_bin, since during the call to pbr_bin the partial results from the threads were being combined. The net effect of multithreading is that the calls to pbr_kernel were now happening concurrently while communication is reduced significantly. The sketch of the thread main function (pbra_grid) is shown below.

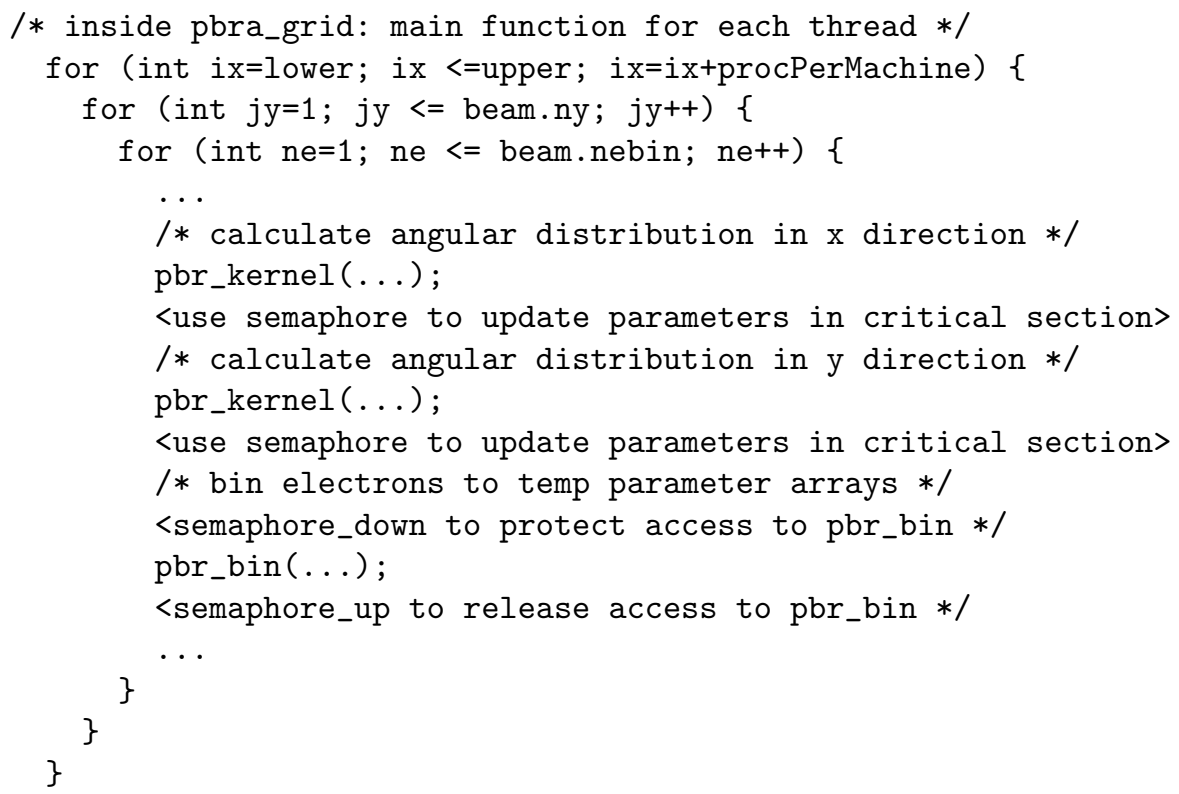

Compared to the sequential program, the multi-threaded version running on one machine with 2 CPUs took 1434 seconds, for a speedup of 1.43. When we ran the PVM program with multi-threading on 12 CPUs, we got a time of 550 seconds for a speedup of 3.73 , about a $20 \%$ improvement. 


\subsection{Adaptive Load Balancing}

Using XPVM and with the help of code instrumentation, it was discovered that although each process had an equal amount of data, the amount of time required is not distributed equally. Also, the uneven distribution had an irregular pattern that varies with each outer iteration. A general load balancing scheme is described below to deal with the unbalanced workloads.

Each slave process sends the time taken for the last outer iteration to the master. Suppose that these times are $t_{0}, t_{1}, t_{2}, \ldots, t_{n-1}$ for $n$ processes. The master computes the average time, say $t_{a v g}$. Based on that, process $P_{i}$ needs to retain a fraction $t_{\text {avg }} / t_{i}$ for the next round. We are using the past iteration to predict the times for the next iteration. We also made the frequency of load balancing be a parameter; that is, whether to load-balance every iteration or every second iteration and so on. The following code shows a sketch of the main function for the slave processes after incorporating the load-balancing.

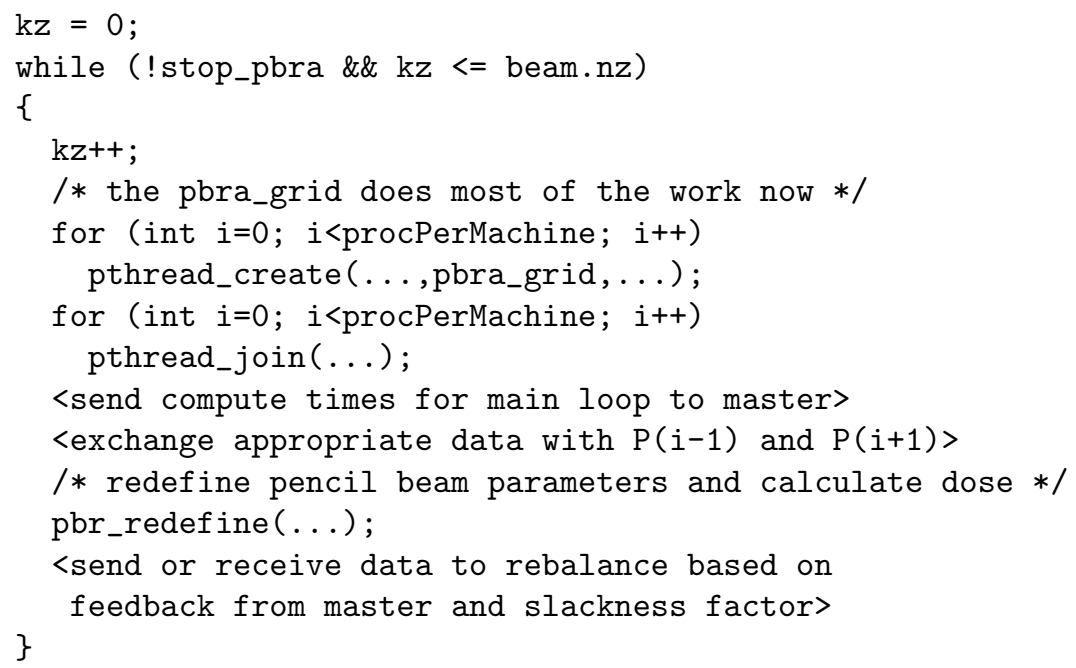

Table 1 shows the effect of load balancing frequency on the parallel runtime.

The next improvement comes from the observation that if a process finishes very fast, it might receive too much data for the next round. The slackness factor allows us to specify a percentage to curtail this effect. If it is set to $80 \%$, then the process receives only $80 \%$ of the data specified by the load-balancing scheme. This allows the data received to be limited but still enables receiving a fair amount to be load-balanced. We experimented with various slackness factors: $90 \%, 80 \%, 70 \%, 60 \%, 50 \%$. A slack factor of $80 \%$ gave an additional $5 \%$ improvement in runtime.

Finally, Table 2 shows the runtime and speedup for the sample data set with all the above refinements in place. The load balancing frequency was set to 4 and the slackness factor was $80 \%$. 
Table 1. Effect of load balancing frequency on runtime for parallel PBRA code running on 6 machines with 2 CPUs each

\begin{tabular}{lr}
\hline $\begin{array}{l}\text { Load Balancing } \\
\text { Frequency }\end{array}$ & $\begin{array}{r}\text { Runtime } \\
\text { (seconds) }\end{array}$ \\
\hline none & 550 \\
1 & 475 \\
2 & 380 \\
3 & 391 \\
4 & 379 \\
5 & 415 \\
\hline
\end{tabular}

Table 2. Parallel runtime versus number of CPUs on a sample data set

\begin{tabular}{lrr}
\hline CPUs & Runtime (secs) & Speedup \\
\hline 1 & 2050 & 1.00 \\
2 & 1434 & 1.42 \\
4 & 1144 & 1.79 \\
6 & 713 & 2.87 \\
8 & 500 & 4.00 \\
10 & 405 & 5.06 \\
12 & 369 & 5.56 \\
\hline
\end{tabular}

\section{Further Results and Discussion}

Table 3. Comparison of various refinements to parallel PBRA program. All times are for 12 CPUs

\begin{tabular}{lrr}
\hline Technique & Time (seconds) & Speedup \\
\hline Sequential & 2050 & 1.00 \\
Partitioning with PVM & 657 & 3.12 \\
Multithreading + PVM & 550 & 3.73 \\
Load balancing + Multithreading + PVM & 369 & 5.56 \\
\hline
\end{tabular}

Table 3 summarizes the improvements obtained with the various refinements on one sample data set.

Table 4 shows the runtime and speedup for six additional data sets. The first column shows the density of the matter through which the beam is traveling. Note that when the density of the matter is high, the electron pencil-beam stops early and thus the computation time is smaller. When the density is low, then the beam goes farther but does not scatter as much, which would also tend 
Table 4. Parallel runtime and speedups for different data sets. The density column shows the density of the matter through which the beam is traveling. All times are for 12 CPUs

\begin{tabular}{lrrr}
\hline $\begin{array}{l}\text { Density } \\
\left(\mathrm{gm} / \mathrm{cm}^{3}\right)\end{array}$ & $\begin{array}{r}\text { Sequential } \\
\text { (seconds) }\end{array}$ & $\begin{array}{r}\text { Parallel } \\
\text { (seconds) }\end{array}$ & Speedup \\
\hline 0.5 & 1246 & 131 & 9.51 \\
0.7 & 2908 & 352 & 8.26 \\
0.9 & 3214 & 364 & 8.83 \\
1.1 & 2958 & 370 & 7.99 \\
1.3 & 2641 & 321 & 8.22 \\
1.5 & 2334 & 300 & 7.78 \\
\hline
\end{tabular}

to reduce the time. Human tissue has density values close to 1.0, which is the density in the sample data set used in the previous section. The average speedup for all the data sets tested was around 8 . The load balancing frequency was set to 4 and the slackness factor was $80 \%$.

Although the speedups obtained are encouraging, further work remains to be done. We would like to test the parallel PBRA program on a larger cluster. Further tuning of the parallel code could lead to more speedups. Testing on a wider variety of data sets should allow us to further customize the load balancing frequency and the slackness factor.

\section{Conclusions}

We have presented a parallelization of electron Pencil Beam Redefinition Algorithm program originally written by Robert Boyd. The program was difficult to parallelize because of the large amounts of data that is accessed in an irregular pattern, requiring varying amounts of time in each iteration. We were able to obtain speedups in the range of 5.56 to 9.5 using 12 CPUs. The speedups obtained were based on three techniques: (1) using a simple partitioning scheme but compensating for the irregularity of data with an interesting data exchange technique that attempts to minimize the amount of data traffic on the network; (2) using threads in conjunction with PVM to reduce communication time for SMP machines; (3) using an adaptive load-balancing technique.

\section{References}

1. K. C. Tsien, "The application of automatic computing machines to radiation treatment," Brit. J. Radiology 28:432-439, 1955.

2. A. S. Shiu and K. R. Hogstrom, "Pencil-beam redefinition algorithm for electron dose distributions," Med. Phys. 18: 7-18, 1991.

3. PVM Home Page: http://www.csm.ornl.gov/pvm/pvm_home.html 